Published in final edited form as:

Chembiochem. 2017 February 16; 18(4): 363-367. doi:10.1002/cbic.201600676.

\title{
OleD Loki as a Catalyst for Tertiary Amine and Hydroxamate Glycosylation
}

\author{
Ryan R. Hughes ${ }^{a}$, Dr. Khaled A. Shaaban ${ }^{a}$, Dr. Jianjun Zhang ${ }^{a}$, Dr. Hongnan Cao ${ }^{b}$, Prof. \\ George N. Phillips Jr. ${ }^{b}$, and Prof. Jon S. Thorson ${ }^{a}$ \\ ${ }^{a}$ Center for Pharmaceutical Research and Innovation, College of Pharmacy, University of \\ Kentucky, 789 South Limestone Street, Lexington, KY 40536 (USA) \\ ${ }^{b}$ Department of Chemistry, Rice University, P. O. Box 1892, MS 60, Houston, TX 77251 (USA)
}

\section{Abstract}

We describe the ability of an engineered glycosyltransferase (OleD Loki) to catalyze the Nglycosylation of tertiary-amine-containing drugs and trichostatin hydroxamate glycosyl ester formation. As such, this study highlights the first bacterial model catalyst for tertiary-amine $\mathrm{N}$ glycosylation and further expands the substrate scope and synthetic potential of engineered OleDs. In addition, this work could open the door to the discovery of similar capabilities among other permissive bacterial glycosyltransferases.

\section{Graphical Abstract}

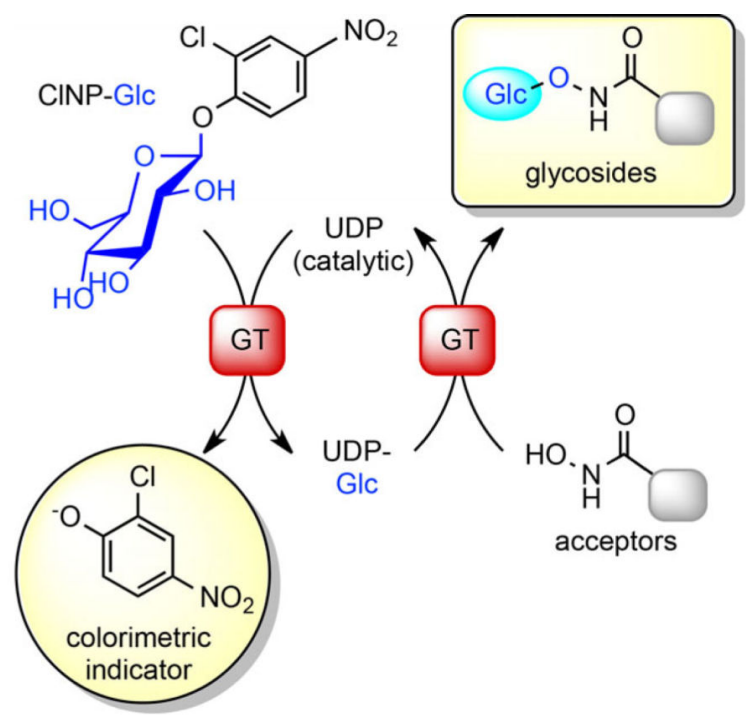

Bacterial model catalyst: The use of a high-throughput colorimetric screen to identify new substrates revealed the engineered macrolide glycosyltransferase OleD Loki as the first reported

Correspondence to: Jon S. Thorson.

Supporting information for this article can be found under: http://dx.doi.org/10.1002/cbic.201600676. 
bacterial glycosyltransferase capable of tertiary-amine $\mathrm{N}$-glycosylation. This study also revealed the same enzyme to catalyze trichostatin hydroxamate glycosyl ester formation.

\section{Keywords}

antidepressants; drug metabolism; glucuronidation; glycosyltransferases; trichostatin

Glycosyltransferases (GTs), among which sugar nucleotide-dependent GTs (also referred to as Leloir GTs) are the most prevalent, mediate the regio- and stereospecific glycoconjugation of diverse sugars to a broad range of acceptors. ${ }^{[1]}$ Although Leloir GTcatalyzed formation of O-glycosides is the most common, corresponding microbial GTs involved in the biosynthesis of $\mathrm{C}_{-},{ }^{[2]} \mathrm{S}-,{ }^{[3]}$ and $\mathrm{N}$-glycosides have also been characterized. Biochemically characterized representative native N-GTs include the indole N-modifying GTs involved in the biosynthesis of indolocarbazoles (AtmG, RebG, NokL, and StaG), ${ }^{[4]}$ the streptothricin guanidino N-modifying GT StnG ${ }^{[5]}$ and bacterial protein/ansamitocin asparagine side chain amide-modifying N-GTs (HMWC, NGT, and Asm25). ${ }^{[6]}$ The gene encoding the putative mannopeptimycin guanidino N-modifying GT has also been reported, ${ }^{[7]}$ and recent studies of the macrolide-inactivating O-GT OleD ${ }^{[8]}$ and corresponding engineered/evolved variants, revealed OleD-catalyzed $\mathrm{N}$-glucosylation of model aromatic/benzyl primary and secondary amines and alkoxyamines. ${ }^{[9]}$ Although examples of tertiary-amine N-glucuronidation of drugs have been reported as part of phase II metabolism, ${ }^{[10]}$ to the best of our knowledge, bacterial/fungal comparators are unprecedented. Within this context, herein we describe the discovery that engineered OleDs can catalyze the $\mathrm{N}$-glycosylation of a set of model tertiary-amine-containing drugs and the formation of hydroxamate glycosyl esters. As such, the work put forth further expands the substrate scope and synthetic potential of enhanced OleDs and presents a complement to conventional synthetic strategies to access quaternary amino- $\mathrm{N}$-glycosides. ${ }^{[11]}$

Inspired by the general reversibility of GT-catalyzed reactions, ${ }^{[12]}$ we recently used simple aromatic glycosides as efficient donors in GT-catalyzed sugar nucleotide synthesis and coupled transglycosylation reactions. ${ }^{[9 a, 13]}$ Importantly, the use of 2-chloro-4-nitrophenyl (CINP) glycoside donors in this context also offered a convenient colorimetric screen to enable the directed evolution of enhanced GTs with broad substrate permissivity and the identification of new GT substrates (Figure $1 \mathrm{~A}$ ). ${ }^{[9 \mathrm{a}]}$ Using CINP- $\beta$-D-Glc and the OleD variant Loki, ${ }^{[9 a]}$ this colorimetric assay was applied to a panel of 28 representative aliphatic tertiary-amine-containing drugs (Figure S1 the Supporting Information). Assays [1 mm putative acceptor, $2 \mathrm{~mm}$ CINP- $\beta$-D-Glc, $0.1 \mathrm{~mm}$ UDP, $25 \mathrm{~mm}$ Tris (pH 8.0), $5 \mathrm{~mm} \mathrm{MgCl}_{2}$, $0.25 \mu \mathrm{M}$ OleD Loki, $20 \mu \mathrm{L}$ total volume, $30^{\circ} \mathrm{C}, 8 \mathrm{~h}$ ] were conducted in triplicate in 384-well plates, and progress was monitored through the change in absorbance at $410 \mathrm{~nm}\left(\Delta A_{410}\right)$. Each plate also contained positive (4-methylumbeliferone; 4-MeUmb) ${ }^{[14]}$ and negative (no acceptor, DMSO) comparator controls. Nine putative primary hits were identified $\left(\Delta A_{410}>2\right.$ standard deviations above the negative control) in this first-phase screen. Reaction mixtures identified as hits were subsequently subjected to HPLC, desalted, ${ }^{[15]}$ and analyzed by LCMS to confirm or refute glycoside formation. This streamlined strategy revealed eight tertiary amines as validated OleD substrates (Figure $1 \mathrm{~B}$ ), none of which contained 
prototypical GT acceptor O-, S-, or N-nucleophiles. To elucidate the nature of the glycosides formed, substrates $\mathbf{1}$ and $\mathbf{2}$ were selected for subsequent scale-up and full structure elucidation based on relative turnover in analytical scale reactions (Figure 1C).

Scaled-up reactions for the chemoenzymatic synthesis of the $\mathbf{1}$ and $\mathbf{2}$ glucosides were conducted in a total volume of $30 \mathrm{~mL}$ [ $1 \mathrm{~mm}$ aglycon, 2 mм CINP- $\beta$-D-Glc, $0.1 \mathrm{~mm}$ UDP, 25 mм Tris (pH 8.0), $5 \mathrm{~mm} \mathrm{MgCl}_{2}, 0.25 \mu \mathrm{m}$ OleD Loki, $30^{\circ} \mathrm{C}, 24 \mathrm{~h}$ ]. Reaction progress was monitored in real time through $\Delta A_{410}$, and upon completion, reactants and products were captured by XAD-16 solid-phase extraction, and the resulting glucosides were subsequently purified by HPLC and size-exclusion chromatography. The molecular formulae of the corresponding products were established as $\mathrm{C}_{23} \mathrm{H}_{30} \mathrm{ClN}_{2} \mathrm{O}_{5} \mathrm{~S}$ and $\mathrm{C}_{25} \mathrm{H}_{34} \mathrm{ClN}_{2} \mathrm{O}_{5}$ by HRESIMS; these are consistent with the glucosylation of $\mathbf{1}$ and $\mathbf{2}$, respectively. Interestingly, ${ }^{1} \mathrm{H}$ and ${ }^{13} \mathrm{C}$ NMR spectra of $\mathbf{1 a}$ and $\mathbf{2 a}$ also revealed signatures that are consistent with atypical glucoside formation. Closer analysis revealed $3^{\prime}-\mathrm{CH}_{2}, 4^{\prime}-\mathrm{CH}_{3}$, and $5^{\prime}-\mathrm{CH}_{3}{ }^{1} \mathrm{H}$ and ${ }^{13} \mathrm{C}$ NMR chemical shifts and clear HMBC correlations that were consistent with quaternary aminoglucosides $3^{\prime}-N-\beta$-D-glucosylchlorpromazine (1a) and $3^{\prime}-N-\beta$-Dglucosylclomipramine (2a; Scheme 1, Tables S1 and S2). Additional COSY and HMBC correlations (Figure S5) were also consistent with this assignment.

The discovery that OleD Loki could catalyze tertiary-amine N-glucosylation prompted a reassessment of additional tertiary-amine-bearing substrates previously identified as OleD Loki substrates-the naturally occurring HDAC inhibitor trichostatin A (9); ${ }^{[16]}$ the c-Raf inhibitor ZM $336372(\mathbf{1 0})^{[17]}$ and the macrolide antibiotic timulcosin (11; Scheme 2) ${ }^{[18]}$ through colorimetric screening and LC-MS. ${ }^{[9 a]}$ For these selected targets, the strategies employed for reaction scale-up, product isolation, and characterization paralleled those described for 1a and 2a. The molecular formulae of the purified glycosides were established by HR-ESIMS to be $\mathrm{C}_{23} \mathrm{H}_{32} \mathrm{~N}_{2} \mathrm{O}_{8}, \mathrm{C}_{29} \mathrm{H}_{33} \mathrm{~N}_{3} \mathrm{O}_{8}$, and $\mathrm{C}_{52} \mathrm{H}_{90} \mathrm{~N}_{2} \mathrm{O}_{18}$; these are consistent with the glucosylation of $\mathbf{9}, \mathbf{1 0}$, and 11, respectively. Unlike for $1 \mathbf{a}$ and $\mathbf{2 a}$, the ${ }^{1} \mathrm{H}$ and ${ }^{13} \mathrm{C}$ NMR spectra of the isolated glucosides revealed signatures that were consistent with Oglucoside formation. Consistent with this, 1D and 2D NMR data revealed 9a to be the unique glucopyranosyl hydroxamate trichostatin $\mathrm{C}$ and notably highlight the first one-step synthesis of this previously reported rare natural product. ${ }^{[19]}$ In a similar fashion, the $1 \mathrm{D}$ and 2D NMR data revealed 10a to be 4- $O-\beta$-D-glucosyl-ZM336372 and 11 a to be $2^{\prime}-O-\beta$-Dglucosyltilmicosin, the latter is consistent with the native activity of wtOleD as a macrolideinactivating glucosyltransferase. ${ }^{[\mathrm{b}]}$

To the best of our knowledge, this study highlights the first reported example of a microbial GT that is capable of catalyzing tertiary-amine $\mathrm{N}$-glycosylation. A comparison of the kinetic parameters reveals that the catalytic competencies of tertiary amine $\mathbf{1}\left(k_{\mathrm{cat}} / K_{\mathrm{m}}=2.2 \times 10^{-4}\right.$ $\left.\mu^{-1} \mathrm{~s}^{-1}\right)$ and hydroxamate $9\left(k_{\mathrm{cat}} / K_{\mathrm{m}}=1.4 \times 10^{-4} \mu \mathrm{m}^{-1} \mathrm{~s}^{-1}\right)$ rival that of the parental OleD Loki acceptor 4-MeUmb $\left(k_{\text {cat }} / K_{\mathrm{m}}=2.2 \times 10^{-4} \mu \mathrm{m}^{-1} \mathrm{~s}^{-1}\right)$. Although tertiary-amine Nglucuronidation has been reported in the context of phase II metabolism, ${ }^{[10]}$ fundamental biochemical study of the corresponding glucuronsyltransferases has been hampered by limited access to suitable in vitro models. Likewise, although sugar conjugation is known to influence small-molecule mechanisms, potency, and ADMET, ${ }^{[1 \mathrm{a}, \mathrm{e}, 20]}$ a lack of practical synthetic or chemoenzymatic access has limited studies to probe the fundamental properties 
of quaternary $\mathrm{N}$-glycosides. ${ }^{[10,11]}$ Our discovery offers a convenient new model for GTcatalyzed tertiary-amine $\mathrm{N}$-glycosylation and a potential complementary synthetic platform for efficient one-step synthesis of quaternary $\mathrm{N}$-glycosides. In a similar fashion, the discovery that OleD Loki is an efficient catalyst for hydroxamate glycosyl ester synthesis further extends the demonstrated synthetic utility of this permissive catalyst. Analysis of the established substrates 1-8 highlights a common alkyl N,N-dimethyl acceptor nucleophile that extends from a hydrophobic aromatic core reminiscent of the longer unsaturated spacer separating the 9 pharmacophore and warhead or the previously reported ability of OleD ASP to catalyze asymmetric 4 '-O-glycosylation of one "arm" of mitoxantrone. ${ }^{[21]}$ The lack of detectable tertiary-amine $\mathrm{N}$-glycosylation with substrates 9-11 may be attributed to substrate orientation in the enzyme-bound complex (as suggested by the determined OleD:macrolide complex, Figure 2) and/or poor nucleophilicity (as anticipated in the context of the $\mathrm{N}, \mathrm{N}$ dimethyl aniline moiety of $\mathbf{9}$ and 10). ${ }^{[22]}$ Although these examples suggest that certain substrate specificity features might infringe on OleD Loki-catalyzed tertiary-amine reactant scope, the underlying discovery also importantly implicates $N, N$-dimethylamino- and/or hydroxamate-functionalized small molecules as a potential acceptors to evaluate in the context of other permissive GTs. ${ }^{[23]}$

\section{Supplementary Material}

Refer to Web version on PubMed Central for supplementary material.

\section{Acknowledgments}

This work was supported, in part, by National Institute of Health grants R37 AI52218 (J.S.T.) and U01 GM098248 (G.N.P.), the National Center for Advancing Translational Sciences (UL1TR001998), the University of Kentucky College of Pharmacy and the University of Kentucky Center for Pharmaceutical Research and Innovation.

\section{References}

1. a) Elshahawi SI, Shaaban KA, Kharel MK, Thorson JS. Chem. Soc. Rev. 2015; 44:7591-7697. [PubMed: 25735878] b) Gloster TM. Curr. Opin. Struct. Biol. 2014; 28:131-141. [PubMed: 25240227] c) Breton C, Fournel-Gigleux S, Palcic MM. Curr. Opin. Struct. Biol. 2012; 22:540-549. [PubMed: 22819665] d) Chang A, Singh S, Phillips GN Jr, Thorson JS. Curr. Opin. Biotechnol. 2011; 22:800-808. [PubMed: 21592771] e) Gantt RW, Peltier-Pain P, Thorson JS. Nat. Prod. Rep. 2011; 28:1811-1853. [PubMed: 21901218] f) Palcic MM. Curr. Opin. Chem. Biol. 2011; 15:226233. [PubMed: 21334964] g) Roychoudhury R, Pohl NL. Curr. Opin. Chem. Biol. 2010; 14:168173. [PubMed: 20129812] h) Lairson LL, Henrissat B, Davies GJ, Withers SG. Annu. Rev. Biochem. 2008; 77:521-555. [PubMed: 18518825] i) Bowles D, Lim EK, Poppenberger B, Vaistij FE. Annu. Rev. Plant Biol. 2006; 57:567-597. [PubMed: 16669774]

2. a) Chen D, Chen R, Wang R, Li J, Xie K, Bian C, Sun L, Zhang X, Liu J, Yang L, Ye F, Yu X, Dai J. Angew. Chem. Int. Ed. 2015; 54:12678-12682. Angew. Chem. 2015, 127, 12869-12873. b) Foshag D, Campbell C, Pawelek PD. Biochim. Biophys. Acta Proteins Proteomics. 2014; 1844:1619_ 1630.c) Gutmann A, Krump C, Bungaruang L, Nidetzky B. Chem. Commun. 2014; 50:54655468.d) Li L, Wang P, Tang Y. J. Antibiot. 2014; 67:65-70. [PubMed: 24022606] e) Wang F, Zhou M, Singh S, Yennamalli RM, Bingman CA, Thorson JS, Phillips GN Jr. Proteins. 2013; 81:12771282. [PubMed: 23526584] f) Gutmann A, Nidetzky B. Angew. Chem. Int. Ed. 2012; 51:1287912883. Angew. Chem. 2012, 124, 13051-13056. g) Härle J, Günther S, Lauinger B, Weber M, Kammerer B, Zechel DL, Luzhetskyy A, Bechthold A. Chem. Biol. 2011; 18:520-530. [PubMed: 21513888] h) Mittler M, Bechthold A, Schulz GE. J. Mol. Biol. 2007; 372:67-76. [PubMed: 17640665] i) Baig I, Kharel M, Kobylyanskyy A, Zhu L, Rebets Y, Ostash B, Luzhetskyy A, Bechthold A, Fedorenko VA, Rohr J. Angew. Chem. Int. Ed. 2006; 45:7842-7846. Angew. Chem. 
2006, 118, 8006-8010. j) Liu T, Kharel MK, Fischer C, McCormick A, Rohr J. ChemBioChem. 2006; 7:1070-1077. [PubMed: 16795121] k) Fischbach MA, Lin H, Liu DR, Walsh CT. Proc. Natl. Acad. Sci. USA. 2005; 102:571-576. [PubMed: 15598734] 1) Bililign T, Hyun CG, Williams JS, Czisny AM, Thorson JS. Chem. Biol. 2004; 11:959-969. [PubMed: 15271354]

3. Kopycki J, Wieduwild E, Kohlschmidt J, Brandt W, Stepanova AN, Alonso JM, Pedras MS, Abel S, Grubb CD. Biochem. J. 2013; 450:37-46. [PubMed: 23150922]

4. a) Chiu HT, Lin YC, Lee MN, Chen YL, Wang MS, Lai CC. Mol. Biosyst. 2009; 5:1192-1203. [PubMed: 19756309] b) Gao Q, Zhang C, Blanchard S, Thorson JS. Chem. Biol. 2006; 13:733-743. [PubMed: 16873021] c) Sánchez C, Méndez C, Salas JA. Nat. Prod. Rep. 2006; 23:1007-1045. [PubMed: 17119643] d) Zhang C, Albermann C, Fu X, Peters NR, Chisholm JD, Zhang G, Gilbert EJ, Wang PG, Van Vranken DL, Thorson JS. ChemBioChem. 2006; 7:795-804. [PubMed: 16575939] e) Salas AP, Zhu L, Sánchez C, Braña AF, Rohr J, Méndez C, Salas JA. Mol. Microbiol. 2005; 58:17-27. [PubMed: 16164546] f) Hyun CG, Bililign T, Liao J, Thorson JS. ChemBioChem. 2003; 4:114-117. [PubMed: 12512086] g) Sánchez C, Butovich IA, Braña AF, Rohr J, Méndez C, Salas JA. Chem. Biol. 2002; 9:519-531. [PubMed: 11983340] h) Ohuchi T, Ikeda-Araki A, Watanabe-Sakamoto A, Kojiri K, Nagashima M, Okanishi M, Suda H. J. Antibiot. 2000; 53:393403. [PubMed: 10866221]

5. Guo Z, Li J, Qin H, Wang M, Lv X, Li X, Chen Y. Angew. Chem. Int. Ed. 2015; 54:5175-5178. Angew. Chem. 2015, 127, 5264-5267.

6. a) Gawthorne JA, Tan NY, Bailey U-M, Davis MR, Wong LW, Naidu R, Fox KL, Jennings MP, Schulz BL. Biochem. Biophys. Res. Commun. 2014; 445:633-638. [PubMed: 24565833] b) Naegeli A, Michaud G, Schubert M, Lin CW, Lizak C, Darbre T, Reymond JL, Aebi M. J. Biol. Chem. 2014; 289:24521-24532. [PubMed: 24962585] c) Choi KJ, Grass S, Paek S, St Geme JW III, Yeo HJ. PLoS One. 2010; 5:e15888. [PubMed: 21209858] d) Grass S, Lichti CF, Townsend RR, Gross J, St Geme JW III. PLoS Pathog. 2010; 6:e1000919. [PubMed: 20523900] e) Zhao P, Bai L, Ma J, Zeng Y, Li L, Zhang Y, Lu C, Dai H, Wu Z, Li Y, Wu X, Chen G, Hao X, Shen Y, Deng Z, Floss HG. Chem. Biol. 2008; 15:863-874. [PubMed: 18721757]

7. Magarvey NA, Haltli B, He M, Greenstein M, Hucul JA. Antimicrob. Agents Chemother. 2006; 50:2167-2177. [PubMed: 16723579]

8. a) Quirós LM, Aguirrezabalaga I, Olano C, Méndez C, Salas JA. Mol. Microbiol. 1998; 28:11771185. [PubMed: 9680207] b) Hernández C, Olano C, Méndez C, Salas JA. Gene. 1993; 134:139_ 140. [PubMed: 8244027]

9. a) Gantt RW, Peltier-Pain P, Cournoyer WJ, Thorson JS. Nat. Chem. Biol. 2011; 7:685-691. [PubMed: 21857660] b) Gantt RW, Goff RD, Williams GJ, Thorson JS. Angew. Chem. Int. Ed. 2008; 47:8889-8892. Angew. Chem. 2008, 120, 9021-9024. c) Yang M, Proctor MR, Bolam DN, Errey JC, Field RA, Gilbert HJ, Davis BG. J. Am. Chem. Soc. 2005; 127:9336-9337. [PubMed: 15984838]

10. a) Kim HJ, Jeong ES, Seo KA, Shin KJ, Choi YJ, Lee SJ, Ghim JL, Sohn DR, Shin JG, Kim DH. Drug Metab. Dispos. 2013; 41:1529-1537. [PubMed: 23704698] b) Qian MR, Zeng S. Acta Pharmacol. Sin. 2006; 27:623-628. [PubMed: 16626519] c) Green MD, King CD, Mojarrabi B, Mackenzie PI, Tephly TR. Drug Metab. Dispos. 1998; 26:507-512. [PubMed: 9616184] d) Green MD, Tephly TR. Drug Metab. Dispos. 1998; 26:860-867. [PubMed: 9733664] e) Hawes EM. Drug Metab. Dispos. 1998; 26:830-837. [PubMed: 9733660] f) Green MD, Tephly TR. Drug Metab. Dispos. 1996; 24:356-363. [PubMed: 8820428] g) Green MD, Bishop WP, Tephly TR. Drug Metab. Dispos. 1995; 23:299-302. [PubMed: 7628292] h) Lehman JP, Fenselau C, Depaulo JR. Drug Metab. Dispos. 1983; 11:221-225. [PubMed: 6135580] i) Lehman JP, Fenselau C. Drug Metab. Dispos. 1982; 10:446-449. [PubMed: 6128190] j) Chaudhuri NK, Servando OA, Manniello MJ, Luders RC, Chao DK, Bartlett MF. Drug Metab. Dispos. 1976; 4:372-378. [PubMed: 8293] k) Porter CC, Arison BH, Gruber VF, Titus DC, Vandenheuvel WJ. Drug Metab. Dispos. 1975; 3:189-197. [PubMed: 238818]

11. a) Iddon L, Bragg RA, Harding JR, Pidathala C, Basca J, Kriby AJ, Stachulski AV. Tetrahedron. 2009; 65:6396-6402.b) Iddon L, Bragg RA, Harding JR, Stachulski AV. Tetrahedron. 2010; 66:537-541.

12. a) Zhang C, Bitto E, Goff RD, Singh S, Bingman CA, Griffith BR, Albermann C, Phillips GN Jr, Thorson JS. Chem. Biol. 2008; 15:842-853. [PubMed: 18721755] b) Zhang C, Moretti R, Jiang J, Thorson JS. ChemBioChem. 2008; 9:2506-2514. [PubMed: 18798210] c) Zhang C, Fu Q, 
Albermann C, Li L, Thorson JS. ChemBioChem. 2007; 8:385-390. [PubMed: 17262863] d) Zhang C, Albermann C, Fu X, Thorson JS. Am. Chem. Soc. J. 2006; 128:16420-16421.e) Zhang C, Griffith BR, Fu Q, Albermann C, Fu X, Lee IK, Li L, Thorson JS. Science. 2006; 313:12911294. [PubMed: 16946071] f) Minami A, Uchida R, Eguchi T, Kakinuma K. J. Am. Chem. Soc. 2005; 127:6148-6149. [PubMed: 15853301]

13. a) Chen Z, Zhang J, Singh S, Peltier-Pain P, Thorson JS, Hinds BJ. ACS Nano. 2014; 8:8104-8112. [PubMed: 25025628] b) Singh S, Peltier-Pain P, Tonelli M, Thorson JS. Org. Lett. 2014; 16:32203223. [PubMed: 24911465] c) Zhang J, Singh S, Hughes RR, Zhou M, Sunkara M, Morris AJ, Thorson JS. ChemBioChem. 2014; 15:647-652. [PubMed: 24677528] d) Gantt RW, Peltier-Pain P, Singh S, Zhou M, Thorson JS. Proc. Natl. Acad. Sci. USA. 2013; 110:7648-7653. [PubMed: 23610417] e) Peltier-Pain P, Marchillo K, Zhou M, Andes DR, Thorson JS. Org. Lett. 2012; 14:5086-5089. [PubMed: 22984807]

14. Williams GJ, Zhang C, Thorson JS. Nat. Chem. Biol. 2007; 3:657-662. [PubMed: 17828251]

15. Gilar M, Belenky A, Wang BH. J. Chromatogr. A. 2001; 921:3-13. [PubMed: 11461010]

16. Vigushin DM, Ali S, Pace PE, Mirsaidi N, Ito K, Adcock I, Coombes RC. Clin. Cancer Res. 2001; 7:971-976. [PubMed: 11309348]

17. a) Kappes A, Vaccaro A, Kunnimalaiyaan M, Chen H. J. Surg. Res. 2006; 133:42-45. [PubMed: 16603190] b) Van Gompel JJ, Kunnimalaiyaan M, Holen K, Chen H. Mol. Cancer Ther. 2005; 4:910-917. [PubMed: 15956248]

18. a) Inamoto T, Kikuchi K, Iijima H, Kawashima Y, Nakai Y, Ogimoto K. J. Vet. Med. Sci. 1994; 56:917-921. [PubMed: 7865594] b) Zhang N, Tao L, Chang J. Life Sci. J. 2010; 7:107-110.

19. Tsuji N, Kobayashi M. J. Antibiot. 1978; 31:939-944. [PubMed: 711616]

20. a) Calvaresi EC, Hergenrother PJ. Chem. Sci. 2013; 4:2319-2333. [PubMed: 24077675] b) Jones EM, Polt R. Front. Chem. 2015; 3c) Moradi SV, Hussein WM, Varamini P, Simerska P, Toth I. Chem. Sci. 2016; 7:2492-2500.

21. Zhou M, Thorson JS. Org. Lett. 2011; 13:2786-2788. [PubMed: 21528870]

22. a) Ammer J, Baidya M, Kobayashi S, Mayr H. J. Phys. Org. Chem. 2010; 23:1029-1035.b) Ofial AR, Mayr H, Phys J. Org. Chem. 2008; 21:584-595.

23. a) Parajuli P, Pandey RP, Trang NT, Oh TJ, Sohng JK. Carbohydr. Res. 2015; 418:13-19. [PubMed: 26513760] b) Song C, Gu L, Liu J, Zhao S, Hong X, Schulenburg K, Schwab W. Plant Cell Physiol. 2015; 56:2478-2493. [PubMed: 26454881] c) Pandey RP, Parajuli P, Koirala N, Lee JH, Park YI, Sohng JK. Mol. Cells. 2014; 37:172-177. [PubMed: 24599002] d) Pandey RP, Gurung RB, Koirala N, Tuoi LT, Sohng JK. Carbohydr. Res. 2014; 393:26-31. [PubMed: 24893262] e) Ono E, Homma Y, Horikawa M, Kunikane S, Imai H, Takahashi S, Kawai Y, Ishiguro M, Fukui Y, Nakayama T. Plant Cell. 2010; 22:2856-2871. [PubMed: 20693356] f) Blanco G, Patallo EP, Braña AF, Trefzer A, Bechthold A, Rohr J, Méndez C, Salas JA. Cell Chem. Biol. 2001; 8:253-263. 
A)

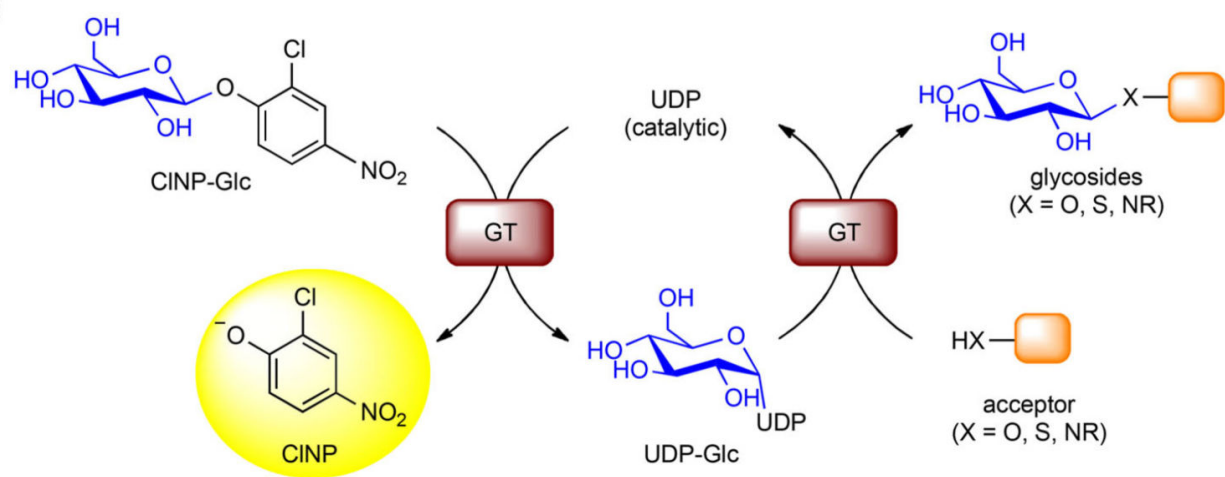

B)<smiles>CN(C)CCCN1c2ccccc2Sc2ccc(Cl)cc21</smiles><smiles>CN(C)CCCN1c2ccccc2CCc2ccccc21</smiles>

5<smiles>CN(C)CCCN1c2ccccc2CCc2ccc(Cl)cc21</smiles>

2<smiles>CN(C)CCC=C1c2ccccc2CCc2ccccc21</smiles>

6<smiles>CN(C)CCC=C1c2ccccc2C=Cc2ccccc21</smiles><smiles>CN(C)CC/C=C1/c2ccccc2COc2ccccc21</smiles>

7<smiles>CN(C)CCCN1c2ccccc2Sc2ccc(C(F)(F)F)cc21</smiles>

C)

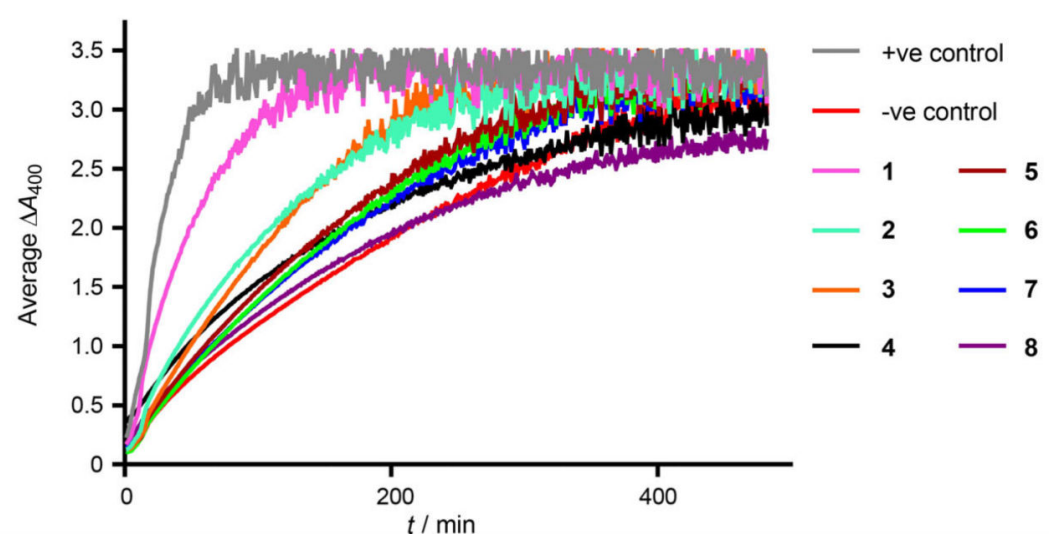

Figure 1.

A) Schematic of colorimetric high-throughput screen. B) Tertiary-amine-containing pharmacophores identified as putative OleD Loki substrates: chlorpromazine (1), clomipramine (2), cyclobenzaprine (3), triflupromazine (4), imipramine (5), amitriptyline (6), doxepin (7), and diltiazem (8). C) Reaction progress as monitored by $\Delta A_{410}$ in the standard $50 \mathrm{~mL}$ assay format [see (A) and main text]. Vehicle without acceptor served as the negative control, and 4-MeUmb served as the positive control. Assays were conducted in triplicate with less than $5 \%$ error between replicates. 


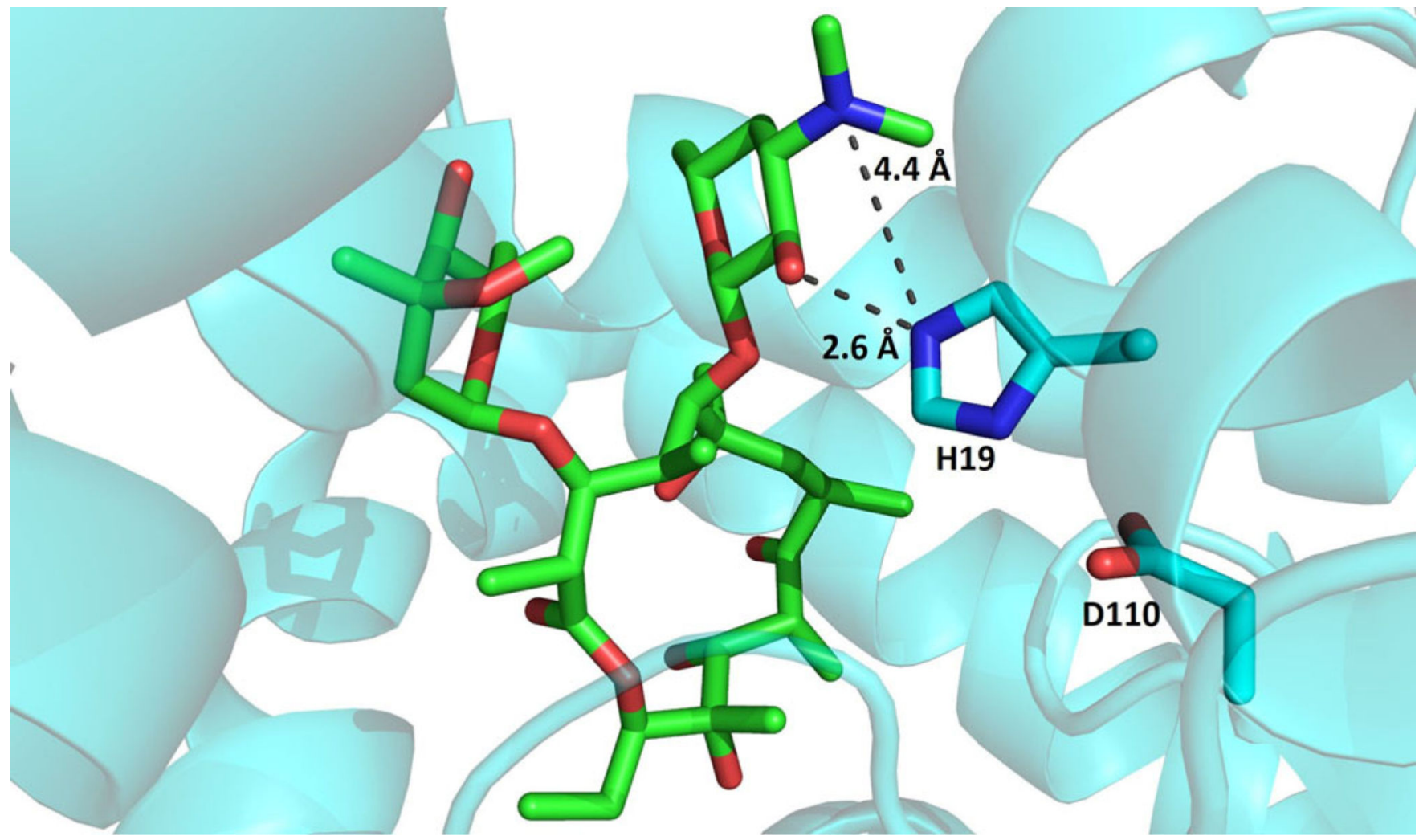

Figure 2.

Crystal structure of wtOleD bound to erythromycin (PDB ID: 2IYF). Dotted lines highlight the proximity of the known acceptor nucleophile (desosamine $2^{\prime}-\mathrm{OH}$, which corresponds to the mycaminose $2^{\prime}-\mathrm{OH}$ in 11) and the closest tertiary amine (desosamine $3^{\prime}-N, N$-dimethyl, which corresponds to the mycaminose $3^{\prime}-N, N$-dimethyl in 11) to the histidine 19 side chain within the active-site H19-D110 acid-base pair. 
<smiles>CCC(C)C(=O)O[Mg]</smiles><smiles>C=C(/C=C\C)NC(=C)/C=C(/C)Cl</smiles>

Scheme 1.

Key HMBC and COSY correlations for compounds 1a and 2a. 

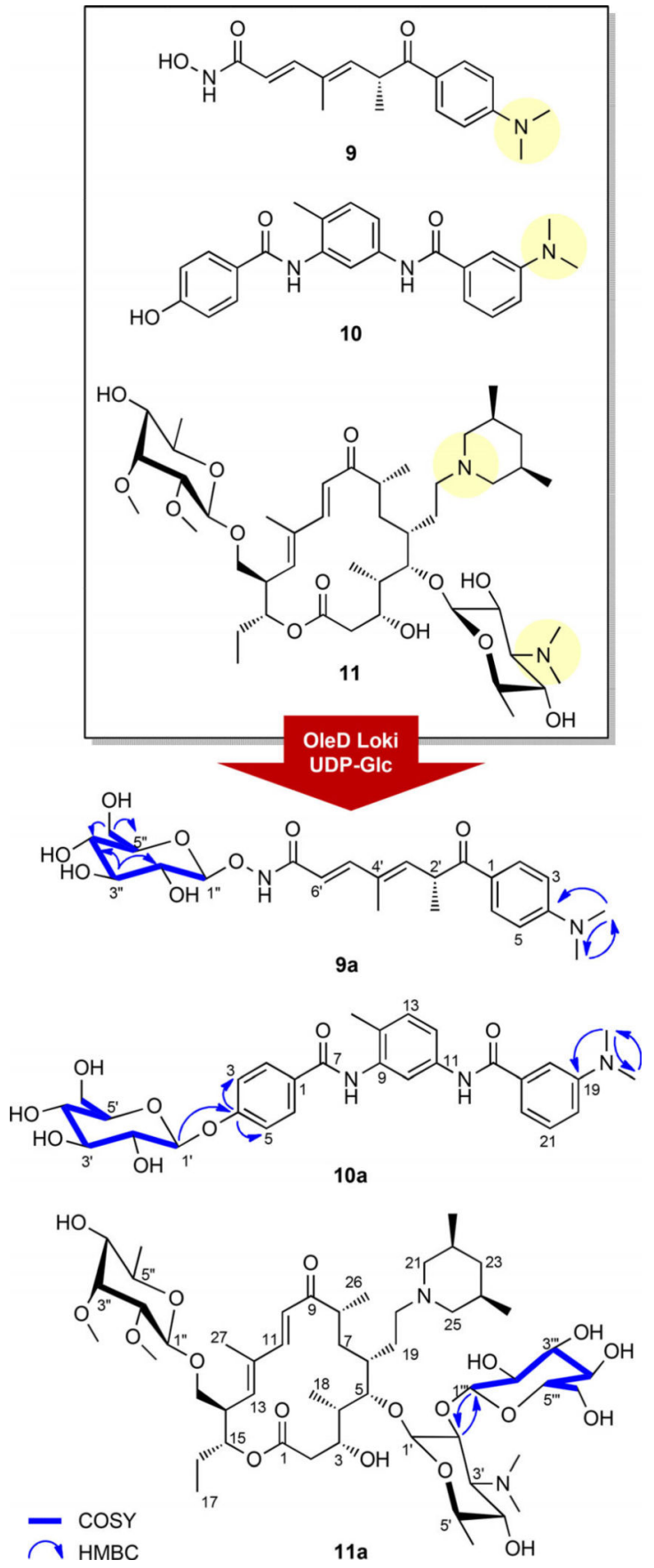

Scheme 2.

Additional tertiary-amine-containing OleD substrates trichostatin A (9), ZM 336372 (10), and tilmicosin (11) and their products. Tertiary amines within 9-11 are shaded yellow, and key HMBC and COSY correlations are highlighted. 